Volume: 2, Issue: 1, January-March 2017, Pages: 105-106, DOI: http://dx.doi.org/10.19082/ah105

\title{
IMPACT OF DATA QUALITY ASSURANCE AND CONTROL SOLUTIONS ON THE COMPLETENESS, ACCURACY, AND CONSISTENCY OF DATA IN A NATIONAL REGISTRY
}

Zahra Azadmanjir ${ }^{1}$, Khatereh Naghdi ${ }^{2}$, Mahdi Safdarian², Aidin Abedi ${ }^{2}$, Mohammad Reza Zarei ${ }^{2}$, Bizhan Aarabi $^{3}$, Seyed Behzad Jazayeri ${ }^{2}$, Mahdi Sharif-Alhosseini ${ }^{2}$, Jalil Arab Kheradmand ${ }^{4}$, Kazem Zendehdel ${ }^{5,6}$, Vafa Rahimi-Movaghar ${ }^{2 *}$

1: Department of Health Information Management, School of Allied Medical Sciences, Tehran University of Medical Sciences, Tehran, Iran

2: Sina Trauma and Surgery Research Center, Tehran University of Medical Sciences, Tehran, Iran

3: Department of Neurosurgery, University of Maryland School of Medicine, Baltimore, Maryland, USA

4: Ahya Neuroscience Research Center, Tehran, Iran

5: Cancer Research Center, Cancer Institute of Iran, Tehran University Medical of Sciences, Tehran, I.R. Iran

6: Office of Disease and Health Outcome Registry, Deputy of Research and Technology, Ministry of Health and Medical Education, I.R Iran

Correspondence:

Vafa Rahimi-Movaghar, MD. Tel: +98216675002, Fax:+982166757009, E-mail: v_rahimi@yahoo.com

Presenter:

Zahra Azadmanjir, PhD

\section{TYPE OF ARTICLE: CONFERENCE ABSTRACT}

\begin{abstract}
Introduction: Data quality is of enormous importance in disease registries as the bases for research, evaluation, and policymaking in health systems. The complexity of data quality assurance and control processes in the registries varies depending on scope, methods of data collection, expertise level of registers, and number of data sources. The aim of the present study was to develop and evaluate a systematic plan to improve data quality for the National Spinal Cord and Column Registry of Iran (NSCIR-IR), which is a multicenter hospital-based registry.

Methods: Our plan to improve data quality included quality assurance (i.e., preventive actions before starting the registry) and quality control (i.e., corrective actions during execution of the registry). Quality assurance was started when determining the data set. We considered availability and accessibility of each data item because it seemed to have a major impact on completeness. In addition, the reliable sources for each data item were specified in the data-gathering guidelines. Other solutions for data quality assurance were considered in designing paper-based case report forms and our software. We designed structured paper forms and minimized them using free text. In addition, to prevent the entry of incorrect data into the software, validation rules were defined and implemented, including 70 semantic rules, 18 syntactic rules, seven temporal rules, and 13 rules for acceptable value range. For quality control, trained staffs were employed as quality reviewers to identify any defect, inaccuracy, and inconsistency of the data. A set of functions was implemented in the software to check data by reviewers, feedback to registrars, and correction. Finally, we evaluated the effects of this plan on the completeness, accuracy, and consistency of our registry data through a seven-month pilot phase.

Results: Completeness was $100 \%$ for 20 socio-demographics data items (e.g., birth date, gender, occupation, etc.), $97 \%$ for national ID and $92.3 \%$ for education level. Completeness was $100 \%$ for admission data $(n=5)$, incident causing injury data including external cause code $(n=17)$, emergency department data $(n=11)$, comorbidity data $(n=9)$, vertebra injury type, level and number $(n=6)$, spinal cord injury data $(n=2)$, other injuries $(n=2)$, interventions $(n=19)$, complications and outcome $(n=13)$, and also American Spinal Cord Association (ASIA) impairment scale $(n=163)$. Completeness was $100 \%$ for all of the emergency medical services data, except arrival and transfer time $(99.43 \%)$ and oxygen saturation $(48.93 \%)$. Consistency was $100 \%$ for all of the
\end{abstract}

\section{Abstracts of First National Congress of Medical Informatics, Mashhad, Iran, February 2017}

(C) 2017 The Authors. This is an open access article under the terms of the Creative Commons Attribution-NonCommercialNoDerivs License, which permits use and distribution in any medium, provided the original work is properly cited, the use is non-commercial and no modifications or adaptations are made. 
data. Accuracy of all data collected by two centers located in Tehran was $100 \%$. Accuracy of vertebra injury type, level and number $(n=6)$, and interventions $(n=19)$ for our collaborating center outside of Tehran was $100 \%$, but it was not evaluable for other data due to lack of access to primary data source.

Conclusion: The solutions for assurance and control of consistency were fully effective. Regarding completeness, our solutions led to a decrease in the missing data. Although designed solutions for the assurance and control of accuracy were effective to registry centers in Tehran, it is necessary to develop an appropriate solution to control the accuracy of the recorded data by centers outside of Tehran.

KEYWORDS: Registries, Quality control, Quality assurance, Heath care, Spinal cord injuries 